\title{
Medizinbibliotheken ${ }^{\text {beta }}$ - Konstant im Wandel
}

\author{
Medical libraries ${ }^{\text {beta }}$ - Change is the constant
}

\begin{abstract}
The focus of the current issue 3/2013 of GMS Medizin - Bibliothek Information is the annual conference 2013 of the German Medical Libraries Association in Berlin. The motto of the conference was "Medical libraries ${ }^{\text {beta }}$ - Change is the constant". The authors in this issue are Michaele Adam (Open access publishing in medicine - in the focus of bibliometrics at the SLUB Dresden), Bruno Bauer, Helmut Dollfuß \& Daniel Formanek (The university library at the Medical University Vienna goes e-only. Change in the provision of literature from p+e to e-only on January ${ }^{\text {st }}, 2013$ ), Henriette Senst \& Jens Erling (The teaching services of the Robert Koch-Institute's library) and Martina Semmler-Schmetz \& Jutta Matrisciano (Medical Libraries ${ }^{\text {beta }}$ - Change is the Constant. Annual meeting 2013 of the German MLA (AGMB), September $16^{\text {th }}$ to $18^{\text {th }}$ in Berlin).

Furthermore this focus issue features articles from Oliver Obst \& Verena Salewsky (How do today's students learn? An e-book study of the branch library of medicine, University of Münster) and from Oliver Obst, Christiane Hofmann, Helge Knüttel \& Petra Zöller ("Ask a question, get an answer, continue your work!" - Survey on the use of UpToDate at the universities of Freiburg, Leipzig, Münster and Regensburg).
\end{abstract}

Keywords: German Medical Libraries Association, annual conference 2013, Berlin, medical library, change, editorial

\section{Zusammenfassung}

Schwerpunktthema der aktuellen Ausgabe 3/2013 von GMS Medizin - Bibliothek - Information ist die Jahrestagung 2013 der Arbeitsgemeinschaft für Medizinisches Bibliothekswesen (AGMB) in Berlin; das Motto lautete "Medizinbibliotheken ${ }^{\text {beta }}$ - Konstant im Wandel“. Die Beiträge der Schwerpunktausgabe wurden verfasst von Michaele Adam (OpenAccess-Publizieren in der Medizin - im Fokus der Bibliometrie an der SLUB Dresden), Bruno Bauer, Helmut Dollfuß \& Daniel Formanek (Universitätsbibliothek der Medizinischen Universität Wien goes e-only: Umstellung des Zeitschriftenbezugs von e-only anstelle von $p+e$ ab 1. Januar 2013), Henriette Senst \& Jens Erling (Das Fortbildungsangebot der Bibliothek des Robert Koch-Instituts: Dienstleistung im Publikationsprozess) und Martina Semmler-Schmetz \& Jutta Matrisciano (Medizinbibliotheken ${ }^{\text {beta }}$ - Konstant im Wandel. Jahrestagung der Arbeitsgemeinschaft für Medizinisches Bibliothekswesen (AGMB) e.V. vom 16.09. bis 18.09.2013 in Berlin).

Weiters bringt die aktuelle Ausgabe von GMS Medizin - Bibliothek Information Beiträge von Oliver Obst \& Verena Salewsky (Wie lernen Studierende heute? E-Book-Umfrage der Zweigbibliothek Medizin der Universität Münster) sowie von Oliver Obst, Christiane Hofmann, Helge Knüttel \& Petra Zöller (,Frage stellen, Antwort bekommen, weiterarbeiten!“ - Umfrage zur Benutzung von UpToDate an den Universitäten Freiburg, Leipzig, Münster und Regensburg).

Schlüsselwörter: Arbeitsgemeinschaft für Medizinisches Bibliothekswesen, Jahrestagung 2013, Berlin, Medizinbibliothek, Veränderung, Editorial

\author{
Bruno Bauer $^{1}$ \\ 1 Medizinische Universität \\ Wien, Universitätsbibliothek, \\ Wien, Österreich
}




\section{AGMB-Jahrestagung 2013 in Berlin}

Ca. 200 Bibliothekarinnen und Bibliothekare folgten der Einladung der Bibliothek der Charité - Universitätsmedizin Berlin (http://bibliothek.charite.de/) und kamen von 16. bis 18. September 2013 an den Tagungsort Campus Virchow-Klinikum. „Medizinbibliotheken ${ }^{\text {beta }}$ - Konstant im Wandel" lautete das Motto der Jahrestagung der Arbeitsgemeinschaft für Medizinisches Bibliothekswesen (AGMB).

Wie bereits in den vergangenen Jahren wurden die Abstracts zu den Vorträgen in Berlin auf der Open-AccessPlattform German Medical Science veröffentlicht [1]; die Präsentationen von sechs der acht Vorträge, die im Plenum bzw. in den Arbeitskreisen präsentiert worden sind, sind über die Website der AGMB (http://www.agmb.de) zugänglich.

- Michaele ADAM (Dresden): „Open Access Publizieren in der Medizin - im Focus der Bibliometrie an der SLUB Dresden“: Abstract [2], Folien [3];

- Gerd ANTES (Freiburg): „Potentielle Rolle der Bibliothekare bei dem Wissenstransfer von der medizinischen Forschung in die Praxis “: Abstract [4];

- Bruno BAUER (Wien): „Aufbau eines Qualitätsmanagementsystems an der Universitätsbibliothek der Medizinischen Universität Wien: erste Zertifizierung gemäß ISO 9001 für eine österreichische Bibliothek “: Abstract [5], Folien [6];

- Bruno BAUER (Wien): „Umstellung des Zeitschriftenbezugs an der Universitätsbibliothek der Medizinischen Universität Wien auf E-Only mit Jahreswechsel 2012/2013“: Abstract [7], Folien [8];

- Jens ERLING \& Henriette SENST (Berlin): „Das Fortbildungsangebot der Bibliothek des Robert Koch-Instituts": Abstract [9], Folien [10];

- Eike HENTSCHEL (Kiel): „Podiumsdiskussion zu E-Books und Aachener Erklärung “: Abstract [11];

- Maxi KINDLING (Berlin): „Digitale Forschungsdaten Status Quo und Perspektiven“: Abstract [12], Folien [13];

- Ulrich KORWITZ (Köln): „Strategie und Markt: Strategieprozess und Ergebnisse der Marktstudie der ZB MED“: Abstract [14];

- Oliver OBST (Münster): „Tablets \& Co: Wie unterstützt die Bibliothek den 'Lernflow' von Digital Natives?": Abstract [15], Folien [16].

Im Rahmen der seit der AGMB-Jahrestagung 2011 ins Programm aufgenommenen Kurzvorträge ( $5+5$ Minuten) wurden in Berlin 6 Themen präsentiert:

- „Google Scholar und Discovery Tools: Der Wunsch der schnellen, umfassenden Suche“ (Martina Gosteli, Zürich);

- „Modernes Computer-Investitions-Programm in der Bibliothek: Weit mehr als ein Ort des stillen Lernens“ (Manuela Roehner, Magdeburg);

- „Auf dem Weg zur nachhaltigen Bibliothek“ (Melanie Kintzel, Hamburg);
- „Professionelles Wachstum durch die EAHIL“ (Oliver Obst, Münster);

- „Catalogue Enrichment und Online-Zeitschriften: zwei Fälle von technologischer Innovation“ (Maurizio Grilli \& Simone Waldboth, Bozen);

- „Buchlieferdienst von der UB Kiel in das Universitätsklinikum Schleswig-Holstein“ (Oliver Weiner, Kiel).

Bereichert wurde das Programm wieder um die vielfältigen Angebote von „Treffpunkt AGMB“, die ausreichend Gelegenheit boten, sich in kleineren Runden über aktuelle Fragestellungen aus der täglichen Praxis von Medizinbibliotheken auszutauschen.

- Treffpunkt 1: „Online-Prüfungstools im Vergleich: AMBOSS, Examen-Online, mediscript“ (Moderation: Stefanus Schweizer, Mainz);

- Treffpunkt 2: „Dienstleistungen im Publikationsprozess: Vertiefung zu den Vorträgen “ (Moderation: Manuela Schulz, Mannheim);

- Treffpunkt 3: „Was ihr wollt! Patron Driven Acquisition (PDA) für E-Books: Nutzerwünsche lenken den Bestandsaufbau“ (Moderation: Jutta Matrisciano, Mannheim);

- Treffpunkt 4a: „Urheberrecht und Medizinbibliotheken“ (Oliver Obst, Münster, \& Christlieb Klages, Berlin);

- Treffpunkt 4b: „Die ZB MED erfindet sich neu: Was wünschen AGMB-Bibliotheken von ihr?" (Elke Roesner, Köln);

- Treffpunkt 5: „MEDPILOT im Einsatz: Offene Fragen und Erfahrungsaustausch“ (Christoph Poley \& Jana Pössel, Köln);

- Treffpunkt 6: „Strategieentwicklung für Bibliotheken“ (Vanessa Proudman, Den Bosch).

\section{Aktuelles Schwerpunktthema: AGMB-Jahrestagung in Berlin 2013}

Die aktuelle Schwerpunktausgabe von GMS MEDIZIN BIBLIOTHEK - INFORMATION bringt vier der bei der AGMBJahrestagung in Berlin gezeigten Präsentationen als Fachbeiträge sowie einen ausführlichen Tagungsbericht.

- Unter dem Titel „Open-Access-Publizieren in der Medizin - im Fokus der Bibliometrie an der SLUB Dresden “ informiert Michaele ADAM (Dresden) über eine Analyse des Teams Bibliometrie der SLUB Dresden. Basierend auf dem Directory of Open Access Journals sowie der in der Datenbank Web of Science (WoS) indexierten Open-Access-Zeitschriften im Bereich der Medizin wurden u.a. ermittelt, dass in ausgewählten WoSFachkategorien ein Open-Access-Anteil von 9,7 Prozent besteht (http://www.egms.de/en/journals/mbi/ 2013-13/mbi000291.shtml).

- Bruno BAUER, Helmut DOLLFUSS und Daniel FORMANEK (Wien) berichten über das Thema „Universitätsbibliothek der Medizinischen Universität Wien goes e-only: Umstellung des Zeitschriftenbezugs von e-only anstelle von p+e ab 1. Januar 2013“. Im Beitrag wer- 
den die Motive für den vollständigen Umstieg des Zeitschriftenbezugs zu einem geplanten Stichtag anhand von Überlegungen und Analysen der Bibliothek zum Nutzerverhalten, zu räumlichen, finanziellen und personellen Ressourcen vorgestellt (http:// www.egms.de/en/journals/mbi/2013-13/ mbi000293.shtml).

- „Das Fortbildungsangebot der Bibliothek des Robert Koch-Instituts: Dienstleistung im Publikationsprozess“ wird im Beitrag von Henriette SENST und Jens ERLING vorgestellt (http://www.egms.de/en/journals/mbi/ 2013-13/mbi000294.shtml).

- Martina SEMMLER-SCHMETZ und Jutta MATRISCIANO (Mannheim) bringen unter dem Titel „Medizinbibliotheken $^{\text {beta }}$ - Konstant im Wandel. Jahrestagung der Arbeitsgemeinschaft für Medizinisches Bibliothekswesen (AGMB) e.V. vom 16.09. bis 18.09.2013 in Berlin" eine ausführliche Nachlese zur abgelaufenen AGMB-Jahrestagung (http://www.egms.de/en/journals/mbi/ 2013-13/mbi000297.shtml).

- Der Beitrag „Aufbau eines Qualitätsmanagementsystems an der Universitätsbibliothek der Medizinischen Universität Wien: erste Zertifizierung gemäß ISO 9001 für eine österreichische Bibliothek " von Bruno BAUER wird aus redaktionellen Gründen erst im kommenden Jahr publiziert werden (http://www.egms.de/en/ journals/mbi/2013-13/mbi000292.shtml).

Weiters bringt die aktuelle Schwerpunktausgabe zwei der drei prämierten Poster, die im Rahmen der AGMB-Jahrestagung in Berlin 2013 präsentiert worden sind:

- Esther PETER-MÜLLER, Anna SCHLOSSER und Martina A. GOSTELI-PETER (Zürich): „iPads für Medizinstudierende - Erfahrungen nach einem halben Jahr Ausleihe: Umfrage an der Medizinbibliothek Careum “ (http://www.egms.de/en/journals/mbi/ 2013-13/mbi000295.shtml);

- Bettina KULLMER (Köln): „Ein Vergleich des Sachstands der Vermittlung von Informationskompetenz (IK) in verschiedenen Disziplinen der Lebenswissenschaften. Ergebnisse einer Online-Befragung von Bibliothekaren universitärer und außeruniversitärer Einrichtungen in Deutschland“; der Beitrag erscheint aus redaktionellen Gründen erst 2014 (http:// www.egms.de/en/journals/mbi/2013-13/ mbi000296.shtml).

Darüber hinaus enthält die aktuelle Ausgabe von GMS MEDIZIN - BIBLIOTHEK - INFORMATION Beiträge zu weiteren Themen, die für Medizinbibliothekarinnen und Medizinbibliothekare von großem Interesse sein sollten.

- "Wie lernen Studierende heute? E-Book-Umfrage der Zweigbibliothek Medizin der Universität Münster“ lautet das Thema des Beitrages von Oliver OBST (Münster) und Verena SALEWSKY (Köln), in dem über bemerkenswerte Ergebnisse einer Benutzerstudie unter Studierenden berichtet wird. 92\% der Studierenden, die an der Umfrage teilgenommen haben, benutzen gedruckte Lehrbücher nahezu immer bzw. oft zum Lernen. Das elektronische Buch hingegen wird von fast der Hälfte der Studierenden regelmäßig genutzt (http:// www.egms.de/en/journals/mbi/2013-13/ mbi000289.shtml).

- Oliver OBST (Münster), Christiane HOFMANN (Leipzig), Helge KNÜTTEL (Regensburg) und Petra ZÖLLER (Freiburg) informieren unter dem Titel „Frage stellen, Antwort bekommen, weiterarbeiten! - Umfrage zur Benutzung von UpToDate an den Universitäten Freiburg, Leipzig, Münster und Regensburg“ über eine Multicenter-Studie, an der 1.083 Ärztinnen und Ärzte, Studierende, Wissenschaftlerinnen und Wissenschaftler und sonstiges medizinisches Fachpersonal an vier deutschen Universitäten teilgenommen haben. Besonders bemerkenswert an den Ergebnissen ist die Tatsache, dass $76 \%$ der befragten Ärztinnen und Ärzte UpToDate nutzen (http://www.egms.de/en/journals/ mbi/2013-13/mbi000290.shtml).

- In der Mitteilung „Aus der AGMB“ informiert Eike HENTSCHEL, Vorsitzender der AGMB, wieder über die jüngste Entwicklung unserer Arbeitsgemeinschaft (http://www.egms.de/en/journals/mbi/2013-13/ mbi000283.shtml).

Breiten Raum widmet die aktuelle Ausgabe von GMS MEDIZIN - BIBLIOTHEK - INFORMATION dem im Vorjahr erstmals vorgestellten Thema Leuchtturmprojekte für Medizinbibliotheken [17].

- Eike HENTSCHEL (Kiel) bringt eine Mitteilung zum Thema „Wettbewerb „Leuchtturmprojekte an Medizinbibliotheken“ 2013: Würdigung der Preisträger“; er informiert über Zielsetzung und Ablauf sowie über die ersten Preisträger des Wettbewerbs (http:// www.egms.de/en/journals/mbi/2013-13/ mbi000284.shtml);

- Maria-Inti METZENDORF stellt „Systematische Literaturrecherche an der Bibliothek der Medizinischen Fakultät Mannheim der Universität Heidelberg“ vor (http://www.egms.de/en/journals/mbi/2013-13/ mbi000286.shtml);

- Helge KNÜTTEL und Gernot DEINZER (Regensburg) präsentieren ihr Konzept für eine „Optimierung des Lehrbuchbestandes durch detaillierte Nutzungsstatistiken" vor (http://www.egms.de/en/journals/mbi/ 2013-13/mbi000285.shtml);

- Stefanus SCHWEIZER informiert über „Konsequente Erschließung und Bewerbung von E-Books in der Bereichsbibliothek Universitätsmedizin (Universitätsbibliothek Mainz)“ (http://www.egms.de/en/journals/mbi/ 2013-13/mbi000287.shtml).

- als Abschluss des Themenblocks Leuchtturmprojekte an Medizinbibliotheken finden Sie die „Ausschreibung für den AGMB-Wettbewerb 2014“ (http:// www.egms.de/en/journals/mbi/2013-13/ mbi000288.shtml).

Ich wünsche Ihnen viel Spaß beim Lesen der aktuellen Ausgabe von GMS MEDIZIN - BIBLIOTHEK - INFORMATION und gute Anregungen für Ihren beruflichen Alltag. 


\section{Literatur}

1. Arbeitsgemeinschaft für medizinisches Bibliothekswesen (AGMB). Jahrestagung der Arbeitsgemeinschaft für medizinisches Bibliothekswesen (AGMB). Berlin, 16.-18.09.2013. Düsseldorf: German Medical Science GMS Publishing House; 2013. Available from: http://www.egms.de/de/meetings/agmb2013/

2. Adam M. Open Access Publizieren in der Medizin - im Focus der Bibliometrie an der SLUB Dresden [Meeting Abstract]. In: Jahrestagung der Arbeitsgemeinschaft für medizinisches Bibliothekswesen (AGMB). Berlin, 16.-18.09.2013. Düsseldorf: German Medical Science GMS Publishing House; 2013. Doc13agmb01. DOI: 10.3205/13agmb01

3. Adam M. Open Access Publizieren in der Medizin - im Focus der Bibliometrie an der SLUB Dresden [Präsentation vom 18.09.2013]. In: Jahrestagung der Arbeitsgemeinschaft für medizinisches Bibliothekswesen (AGMB). Berlin, 16.-18.09.2013. Available from: http://www.agmb.de/ papoopro/dokumente/upload/2662d_agmb_2013_adam.pdf

4. Antes G. Potentielle Rolle der Bibliothekare bei dem Wissenstransfer von der medizinischen Forschung in die Praxis [Meeting Abstract]. In: Jahrestagung der Arbeitsgemeinschaft für medizinisches Bibliothekswesen (AGMB). Berlin,

16.-18.09.2013. Düsseldorf: German Medical Science GMS Publishing House; 2013. Doc13agmb02. DOI: 10.3205/13agmb02

5. Bauer B. Aufbau eines Qualitätsmanagementsystems an der Universitätsbibliothek der Medizinischen Universität Wien: erste Zertifizierung gemäß ISO 9001 für eine österreichische Bibliothek [Meeting Abstract]. In: Jahrestagung der Arbeitsgemeinschaft für medizinisches Bibliothekswesen (AGMB). Berlin, 16.-18.09.2013. Düsseldorf: German Medical Science GMS Publishing House; 2013. Doc13agmb03. DOI: 10.3205/13agmb03

6. Bauer B. Aufbau eines Qualitätsmanagementsystems an der Universitätsbibliothek der Medizinischen Universität Wien: erste Zertifizierung gemäß ISO 9001 für eine österreichische Bibliothek [Präsentation vom 18.09.2013]. In: Jahrestagung der Arbeitsgemeinschaft für medizinisches Bibliothekswesen (AGMB). Berlin, 16.-18.09.2013. Available from: http://www.agmb.de/ papoopro/dokumente/upload/00094_agmb_2013_bauer.pdf

7. Bauer B. Umstellung des Zeitschriftenbezugs an der Universitätsbibliothek der Medizinischen Universität Wien auf EOnly mit Jahreswechsel 2012/2013 [Meeting Abstract]. In: Jahrestagung der Arbeitsgemeinschaft für medizinisches Bibliothekswesen (AGMB). Berlin, 16.-18.09.2013. Düsseldorf: German Medical Science GMS Publishing House; 2013. Doc13agmb04. DOI: 10.3205/13agmb04

8. Bauer B. Umstellung des Zeitschriftenbezugs an der Universitätsbibliothek der Medizinischen Universität Wien auf EOnly mit Jahreswechsel 2012/2013 [Präsentation vom 16.09.2013]. In: Jahrestagung der Arbeitsgemeinschaft für medizinisches Bibliothekswesen (AGMB). Berlin, 16.-18.09.2013. Available from: http://www.agmb.de/ papoopro/dokumente/upload/07199_agmb_2013_bauer_2.pdf

9. Erling J, Senst H. Das Fortbildungsangebot der Bibliothek des Robert Koch-Instituts [Meeting Abstract]. In: Jahrestagung der Arbeitsgemeinschaft für medizinisches Bibliothekswesen (AGMB). Berlin, 16.-18.09.2013. Düsseldorf: German Medical Science GMS Publishing House; 2013. Doc13agmb05. DOI: 10.3205/13agmb05

10. Erling J, Senst H. Das Fortbildungsangebot der Bibliothek des Robert Koch-Instituts [Präsentation vom 17.09.2013]. In: Jahrestagung der Arbeitsgemeinschaft für medizinisches Bibliothekswesen (AGMB). Berlin, 16.-18.09.2013. Available from: http://www.agmb.de/papoopro/dokumente/upload/9fc5d_ rki_präsentation_schulungsangebot_bibliothek.pdf
11. Hentschel E. Podiumsdiskussion zu E-Books und Aachener Erklärung [Meeting Abstract]. In: Jahrestagung der Arbeitsgemeinschaft für medizinisches Bibliothekswesen (AGMB). Berlin, 16.-18.09.2013. Düsseldorf: German Medical Science GMS Publishing House; 2013. Doc13agmb06. DOI: 10.3205/13agmb06

12. Kindling M. Digitale Forschungsdaten - Status Quo und Perspektiven [Meeting Abstract]. In: Jahrestagung der Arbeitsgemeinschaft für medizinisches Bibliothekswesen (AGMB). Berlin, 16.-18.09.2013. Düsseldorf: German Medical Science GMS Publishing House; 2013. Doc13agmb07. DOI: 10.3205/13agmb07

13. Kindling M. Digitale Forschungsdaten - Status Quo und Perspektiven [Präsentation vom 18.09.2013]. In: Jahrestagung der Arbeitsgemeinschaft für medizinisches Bibliothekswesen (AGMB). Berlin, 16.-18.09.2013. Available from: http:// de.slideshare.net/MaxiKindling/digitale-forschungsdaten-statusquo-und-perspektiven\%20

14. Korwitz U. Strategie und Markt: Strategieprozess und Ergebnisse der Marktstudie der ZB MED [Meeting Abstract]. In: Jahrestagung der Arbeitsgemeinschaft für medizinisches Bibliothekswesen (AGMB). Berlin, 16.-18.09.2013. Düsseldorf: German Medical Science GMS Publishing House; 2013. Doc13agmb08. DOI: 10.3205/13agmb08

15. Obst O. Tablets \& Co: Wie unterstützt die Bibliothek den 'Lernflow' von Digital Natives? [Meeting Abstract]. In: Jahrestagung der Arbeitsgemeinschaft für medizinisches Bibliothekswesen (AGMB). Berlin, 16.-18.09.2013. Düsseldorf: German Medical Science GMS Publishing House; 2013. Doc13agmb09. DOI: 10.3205/13agmb09

16. Obst O. Tablets \& Co: Wie unterstützt die Bibliothek den 'Lernflow' von Digital Natives? [Präsentation vom 18.09.2013]. In: Jahrestagung der Arbeitsgemeinschaft für medizinisches Bibliothekswesen (AGMB). Berlin, 16.-18.09.2013. Available from: http://www.agmb.de/papoopro/dokumente/upload/ 8700d_tablets-und-co-final.pdf

17. Hentschel E. Leuchtturmprojekte an Medizinbibliotheken: Ausschreibung für den AGMB-Wettbewerb 2013. GMS Med Bibl Inf. 2012;12(3):Doc18. DOI: 10.3205/mbi000254

\section{Korrespondenzadresse:}

\section{Mag. Bruno Bauer}

Universitätsbibliothek der Medizinischen Universität Wien, Währinger Gürtel 18-20, A-1097 Wien, Tel.: +43 (0) 140160-26100, Fax: +43 (0) 140160-926001 bruno.bauer@meduniwien.ac.at

\section{Bitte zitieren als}

Bauer B. Medizinbibliotheken ${ }^{\text {beta }}$ - Konstant im Wandel. GMS Med Bibl Inf. 2013;13(3):Doc18.

DOI: 10.3205/mbi000282, URN: urn:nbn:de:0183-mbi0002822

Artikel online frei zugänglich unter

http://www.egms.de/en/journals/mbi/2013-13/mbi000282.shtml

Veröffentlicht: 20.12 .2013

\section{Copyright}

(c)2013 Bauer. Dieser Artikel ist ein Open Access-Artikel und steht unter den Creative Commons Lizenzbedingungen (http://creativecommons.org/licenses/by-nc-nd/3.0/deed.de). Er darf vervielfältigt, verbreitet und öffentlich zugänglich gemacht werden, vorausgesetzt dass Autor und Quelle genannt werden. 